\title{
Relationship Between Timing of Peak Height Velocity and Pubertal Staging in Boys and Girls
}

\author{
Andrea Granados1, Achamyeleh Gebremariam2, Joyce M. Lee1,2 \\ 1 University of Michigan Faculty of Medicine, Department of Pediatric Endocrinology, Michigan, United States of America \\ 2 University of Michigan Faculty of Medicine, Child Health Evaluation and Research (CHEAR) Unit, Division of General Pediatrics, \\ Michigan, United States of America
}

\begin{abstract}
Growth and pubertal development are important health markers. We used the data of a longitudinal growth study on a contemporary sample of US youth to examine the relationship between peak height velocity (PHV) and Tanner staging. We observed a substantial variability in the timing of PHV across Tanner stages, which is an important consideration for clinicians when assessing growth.
\end{abstract}

Key words: Peak height velocity, pubertal staging, growth spurt, adolescent growth

Conflict of interest: None declared

Received: 18.03 .2015

Accepted: 08.06 .2015

\section{Introduction}

Linear growth and pubertal development are critical markers of the overall health status of a child. Linear growth occurs in tandem with pubertal development, with the activation of the hypothalamic pituitary gonadal axis as the proposed driver of the "adolescent growth spurt" (1). A better understanding of how pubertal staging correlates with the adolescent growth spurt can help clinicians distinguish systemic disease or endocrine dysfunction (2) from normal variants of growth, such as constitutional delay of growth and maturation (3).

The literature on the relationship between the adolescent growth spurt or peak height velocity (PHV) and pubertal staging is relatively sparse, beyond the initial studies performed by Tanner in the mid 1900's (4). We are unaware of studies that have used contemporary clinical longitudinal cohorts to examine this relationship.

Our objective was to evaluate the relationship between timing of PHV with pubertal development across stages of puberty for boys and girls.

\section{Methods}

The National Institute of Child Health and Human Development (NICHD) Study of Early Child and Youth Development was a longitudinal study of growth and development that followed children from infancy to 15 years of age starting in 1991. The study recruited a total of 1364 children whose birth was normal, uncomplicated and who were born to healthy mothers.

Height and weight were measured by trained research assistants using standardized procedures $(5,6)$. Height and weight measurements were performed up to 11 times throughout the study, at ages $2,3,5,7,9-15$ years. Since not 
Granados A et al.

Growth Velocity and Puberty in Adolescents

all children were measured at all time points, we performed imputations using Stata ICE statistical software. After imputing data, we excluded 616 subjects with more than 2 missing measurements after 6 years of age for girls and 8 years for boys. Additionally, we excluded 346 participants since negative height data were recorded in the imputed data. We included 402 subjects with available information on weight, height and pubertal staging.

We used in our study Tanner stage (TS) data for breast development in girls and for genitalia in boys. The measurements were obtained by pediatric endocrinologist or nurse practitioners using visual inspection and palpation. Menarche data was obtained by maternal report via questionnaire.

$\mathrm{HV}$ was calculated as the increment in height divided by the difference in age between two consecutive measurements at the study visits. PHV was identified as the largest increase in $\mathrm{HV}$ after age 6 years for girls and after age 8 years for boys and the age corresponding to that PHV was tagged as age at PHV.

\section{Results}

Overall there were 402 children, of whom 156 were female and 246 were male. The majority $(80.6 \%)$ of the subjects were classified as white and the remainder $(19.4 \%)$ as nonwhite. At 9 years of age, $70.1 \%$ of the population had a normal weight (defined as a body mass index (BMI) of $5-84 \%)$ and $28.6 \%$ were overweight or obese (defined as a $\mathrm{BMI} \geq 85 \%$ ). The mean age at PHV was 12.1 years (1.4 SD) for females and 13.7 years (1.4 SD) for males. PHV for females was $9.8 \mathrm{~cm} /$ year and for boys was $11.3 \mathrm{~cm} /$ year. Table 1 shows the percentage of children who achieved PHV across all stages of puberty. The majority of girls (69.1\%) had achieved PHV by TS 3 and the majority of boys by TS 4 (58.9\%). Additionally, we found that $70.6 \%$ of girls had attained their PHV by the time of menarche.

\section{Conclusion}

Our study is one of the first to examine the relationship between timing of $\mathrm{PHV}$ and pubertal stage in a recent population-based cohort of healthy children. We were surprised by the substantial variability in the timing of PHV, which suggests that PHV is part of a dynamic process that can be expected to occur in later stages of puberty. Current knowledge about the relationship between PHV and pubertal development was previously based on data provided by Tanner in 1976 (4). Tanner reported the relationship of $\mathrm{PHV}$ with pubertal stage as a correlation and concluded that age at PHV was related to age at TS 2 for breast in girls and TS 3 in boys (4). However this study was based on a UK population of children growing up in the 1950's with a limited sample size (6). More recently, Kelly et al (7) published reference ranges for annual PHV for "earlier", "average" and "later" maturers from a large multicenter, multiethnic contemporary US cohort of children, but the relationship between PHV across all pubertal stages was not evaluated.

Our study can provide reassurance to clinicians and families with concerns for their child's linear growth. For example, a boy who is TS 3 with no evidence of a growth spurt may still have normal growth because the majority of boys do not reach their PHV until TS 4.

There were limitations to our study. First, we had only yearly measurements of height, weight and pubertal status from 9-15 years of age. Second, we did not have information about adult height for all participants since they were only followed up to 15 years of age. Third, TS, although performed by trained nurse practitioners and endocrinologists by palpation and visual inspection, is a somewhat subjective measurement that may lead to TS misclassification. For instance, differentiating between TS 1 and 2 (enlargement of testis and slight thinning and reddening of the scrotal skin) and between TS 4 and 5 (further scrotum enlargement of $12-20 \mathrm{~cm}$ vs. $>20 \mathrm{~cm}$ and darkening) could explain why PHV is observed in our cohort at TS 1 or 5, not typically expected in the clinical setting. Finally, we did not account for factors that may influence linear growth and pubertal timing such as obesity, which can accelerate puberty in girls (8) but may have mixed effects in boys (9).

Table 1. Percentage of children who achieved peak height velocity across all stages of puberty

\begin{tabular}{|l|l|l|l|l|l|l|}
\hline \multirow{2}{*}{ Tanner stage (breast for girls, genitalia for boys) } & \multicolumn{2}{|l|}{ Boys } & \multicolumn{3}{l|}{ Girls } \\
\cline { 2 - 8 } & Mean Age & SD & PHV (\%) & Mean Age & SD & PHV (\%) \\
\hline Tanner stage 1 & - & - & 5.9 & - & - & 6.9 \\
\hline Tanner stage 2 & 11.8 & 1.7 & 12.5 & 10.9 & 1.6 & 29.4 \\
\hline Tanner stage 3 & 13.2 & 0.8 & 30.4 & 12.8 & 1.0 & 69.1 \\
\hline Tanner stage 4 & 13.8 & 0.7 & 58.9 & 13.6 & 0.8 & 92.6 \\
\hline Tanner stage 5 & 14.3 & 0.4 & 70.7 & 14.3 & 0.5 & 95.1 \\
\hline PHV: peak height velocity, SD: standard deviation & & & & \\
\hline
\end{tabular}


Granados A et al.

Growth Velocity and Puberty in Adolescents

This study emphasizes the considerable variation in timing of PHV as it relates to pubertal stage. More empiric studies of growth in contemporary cohorts of children are needed to guide pediatricians in the overall assessment of linear growth and pubertal development.

\section{Acknowledgements}

This study was funded by $\mathrm{NIH}$ grant (NICHD) 1R03HD061438-01A2.

This study was considered exempt and not regulated by the University of Michigan IRB.

\section{References}

1. Ebling FJ. The neuroendocrine timing of puberty. Reproduction 2005;129:675-683.

2. Rosenfield RL. Essentials of growth diagnosis. Endocrinol Metab Clin North Am 1996;25:743-758.
3. Rogol AD, Hayden GF. Etiologies and early diagnosis of short stature and growth failure in children and adolescents. J Pediatr 2014;164(Suppl 5):1-14.

4. Tanner JM. Growth of the human at the time of adolescence. Lect Sci Basis Med 1953;1:308-363.

5. Marshall WA, Tanner JM. Variations in pattern of pubertal changes in girls. Arch Dis Child 1969;44:291-303.

6. Tanner JM, Davies PS. Clinical longitudinal standards for height and height velocity for North American children. $J$ Pediatr 1985;107:317-329.

7. Kelly A, Winer KK, Kalkwarf H, Oberfield SE, Lappe J, Gilsanz $\bigvee$, Zemel BS. Age-based reference ranges for annual height velocity in US children. J Clin Endocrinol Metab 2014;99:2104-2112. Epub 2014 Mar 6

8. Lee JM, Appugliese D, Kaciroti N, Corwyn RF, Bradley RH, Lumeng JC. Weight status in young girls and the onset of puberty. Pediatrics 2007;119:624-630.

9. Lee JM, Kaciroti N, Appugliese D, Corwyn RF, Bradley RH, Lumeng JC. Body mass index and timing of pubertal initiation in boys. Arch PediatrAdolesc Med 2010;164:139-144. 